\title{
Fermat's last theorem, a proof based to limit
}

\begin{abstract}
Fermat's Last Theorem is the most famous mathematical problem of all times. It has never stopped being a challenge for the broader mathematical community, mainly because Wile's proof $^{1}$ was based on an extensive mathematical background that was not nearly available in Fermat's era.

In the present work we assume that the Theorem states a true proposition and we end up in a contradiction, proving the theorem holds for large values of $\mathrm{n}$. The method is based on the general concept of the limit as it was presented in the recent work of A.Mazaris. ${ }^{2}$

This partial proof, in addition to its mathematical and historical value, has another special feature: it is a very brief proof of a problem that has dealt with the international mathematical community for centuries. The simplicity of this approach leaves room for us to include the possibility that this could be close to the line of thinking Fermat himself used when he stated that has come up with a short proof of his proposal.
\end{abstract}

Volume I Issue 4 - 2018

\section{Serbi L}

University of Athens, Greece

Correspondence: Serbi L, Gnosis Mathematical Research Institute, 25, Agiou Antoniou str, 15235 Vrilissia, Greece, Tel 3069 4002 5916,Email a.mazaris@yahoo.gr

Received: June 26, 2018 | Published: July 16, 2018

Keywords: fermat, fermat's last theorem, diophantines equations

\section{Introduction}

Has Fermat proved his last theorem as he claimed in his writings? This is a question of both historical and mathematical value. In this study, we partially prove Fermat's last theorem, based on the concept of limit that was known in considerable depth back in the 16th century. We provide a short prove that is valid for large values of $n$, suggesting that it could have been close to Fermat's unrevealed proof.

The idea of the proof follows (but does not depend on) the broader idea presented in Mazaris. ${ }^{2}$ The proof itself can be viewed as partial, in the sense that it does not cover the whole possible range of values for $\mathrm{n}$.

\section{A novel and targeted approach}

Fermat's famous Last Theorem states that: if $a, b, c$ is positive integers then there is no natural integer $n>2$ such that:

$$
a^{n}+b^{n}=c^{n}
$$

We will show that for large values of $n$, Fermat's theorem holds. We will attempt a proof by contradiction and use of the concept of limit, as applied in sequences of natural numbers. We assume that $a$, $b, c, n$ are positive integer numbers $(\mathrm{n}>2)$ that satisfy the Theorem. Obviously each of $a^{n}, b^{n}, c^{n}$ are also positive integers. Dividing equation (1) by $c^{n}$ results:

$$
\left(\frac{a}{c}\right)^{n}+\left(\frac{b}{c}\right)^{n}=1
$$

If we assume that $\mathrm{n}$ takes very large values $(n \rightarrow+\infty)$ and employ the concept of limit since $+\infty$ is accumulation point for positive integer numbers, then equation (2) can be written as:

$$
\lim _{n \rightarrow+\infty}\left(\frac{a}{c}\right)^{n}+\lim _{n \rightarrow+\infty}\left(\frac{b}{c}\right)^{n}=1
$$

We will now prove that each of the two left hand side terms of equation (3) equals 0 , leading to a contradiction $(0+0=1)$. Equation (1) automatically implies that $c^{n}>a^{n}$ and $c^{n}>b^{n}$, thus $c>a$ and $c>b$.

Consider $\varepsilon$ to be an arbitrary small number, such that:

$$
\left(\frac{a}{c}\right)^{n}<\varepsilon \text { and }\left(\frac{b}{c}\right)^{n}<\varepsilon
$$

Then $\ln \left(\frac{a}{c}\right)^{n}<\ln \varepsilon$ and $\ln \left(\frac{b}{c}\right)^{n}<\ln \varepsilon$

Since $c>a$ and $c>b$ it follows that

$$
\ln \frac{a}{c}<0 \text { and } \ln \frac{b}{c}<0
$$

and consequently

$$
n>\frac{\ln \varepsilon}{\ln \frac{a}{c}} \text { and } n>\frac{\ln \varepsilon}{\ln \frac{a}{c}}
$$

If $k_{1}$ and $k_{2}$ are the lower-value integers that satisfy

$$
k_{1}>\frac{\ln \varepsilon}{\ln \frac{a}{c}} \text { and } k_{2}>\frac{\ln \varepsilon}{\ln \frac{b}{c}}
$$

Then for every natural number $n>k_{1}$ it is holds

$$
\left(\frac{a}{c}\right)^{n}<\varepsilon
$$

And for every natural number $n>k_{2}$ it is holds

$$
\left(\frac{b}{c}\right)^{n}<\varepsilon
$$

Therefore, both sequences $\left(\frac{b}{c}\right)^{n}$ and $\left(\frac{b}{c}\right)^{n}$ converge to 0 , or

$$
\lim _{n \rightarrow+\infty}\left(\frac{a}{c}\right)^{n}=\lim _{n \rightarrow+\infty}\left(\frac{b}{c}\right)^{n}=0
$$

And thus equation (3) leads to a contradiction $(0+0=1)$. As a result, equation (1) cannot be satisfied, and so Fermat's Theorem is proved for large values of $n . \mathrm{n}$ is a very large (though large) positive integer such that $\mathrm{n}>\max \left\{\mathrm{k}_{1}, \mathrm{k}_{2}\right\}$ were $\mathrm{k}_{1}$ and $\mathrm{k}_{2}$ are the lower - value integers that satisfy $\mathrm{k}_{1}>\frac{\ln \varepsilon}{\ln \frac{\mathrm{a}}{\mathrm{c}}}$ and $k_{2}>\frac{\ln \varepsilon}{\ln \frac{\mathrm{b}}{\mathrm{c}}}$.

The theorem therefore proves for $\stackrel{\mathrm{c}}{\mathrm{c}}$ ery positive integer number $n>\max \left\{k_{1}, k_{2}\right\}$. 


\section{Discussion and conclusion}

Combining the fundamental concept of the limit with a classical proofing method, as proof by contradiction is, we proved Fermat's last theorem for large values of $n$. This line of thinking is based on methods and tools that were available to Fermat. This study does not provide a complete proof of the theorem, as it concerns only a range of values for $\mathrm{n}$, but it retains its mathematical value, putting forward a rather simplistic line of logically connected arguments that lead to a solid conclusion.

\section{Acknowledgements}

None.

\section{Conflict of interest}

The author declares that there is no conflict of interest.

\section{References}

1. Wiles AJ. Modular Elliptic Curves and Fermat's Last Theorem. Annals of Mathematics. 1995;141(3):443-551.

2. Mazaris A. A new proof of Fermat's Last Theorem. OAJMTP. 2018;1(3):107-113. 